\title{
ZEROS OF FUNCTIONS IN THE BERGMAN SPACES
}

\author{
BY CHARLES HOROWITZ
}

Communicated by R. C. Buck, November 6, 1973

A function $f(z)$ analytic in the unit disk is said to belong to the Bergman space $A^{p}(0<p<\infty)$ if $\int_{0}^{1} \int_{0}^{2 \pi}\left|f\left(r e^{i \theta}\right)\right|^{p} r d r d \theta<\infty$. It is clear that $A^{p}$ contains the Hardy space $H^{p}$ of analytic functions for which $\lim _{r \rightarrow 1} \int_{0}^{2 \pi}\left|f\left(r e^{i \theta}\right)\right|^{p} d \theta<$ $\infty$. We adopt the convention that $A^{\infty}=H^{\infty}$, the space of bounded analytic functions in the disc.

Assuming that $f(0) \neq 0$, we list the zeros of $f$ in order of nondecreasing modulus: $0<\left|z_{1}\right| \leqq\left|z_{2}\right| \leqq\left|z_{3}\right| \leqq \cdots<1$. We repeat $z_{i}$ according to the multiplicity of the zero of $f$ at $z_{i}$. The sequence $\left\{z_{i}\right\}$ is called the zero set of $f$. If $f \in A^{p}$ (resp. $H^{p}$ ), then $z_{i}$ will be called an $A^{p}$ (resp. $H^{p}$ ) zero set. It has long been known that $H^{p}$ zero sets $(0<p \leqq \infty)$ are completely characterized by the condition $\prod_{k=1}^{\infty} 1 /\left|z_{k}\right|<\infty$. (Equivalently, $\sum_{k=1}^{\infty} 1-\left|z_{k}\right|<\infty$.) In particular, the condition is independent of $p$. Our results show that the situation for $A^{p}$ zero sets is considerably more complex.

Lemma 1. If $\left\{z_{k}\right\}$ is an $A^{p}$ zero set $(0<p<\infty)$, then

$$
\prod_{K=1}^{N} \frac{1}{\left|z_{k}\right|}=O\left(N^{1 / p}\right) \text {. }
$$

Corollary. If $\left\{z_{k}\right\}$ is an $A^{p}$ zero set $(0<p<\infty)$, then for each $\varepsilon>0$,

$$
\sum_{k=1}^{\infty}\left(1-\left|z_{k}\right|\right)\left\{\log \frac{1}{1-\left|z_{k}\right|}\right\}^{-1-\varepsilon}<\infty .
$$

If $f(z)=\sum_{n=0}^{\infty} a_{n} z^{n}$, let $S_{N}^{(p)}=\sum_{k=1}^{N}\left|a_{k}\right|^{p}, p>0$.

LEMMA 2. If $S_{N}^{(2)}=O\left(N^{\alpha}\right)$ for some $\alpha \geqq 1$, then $f \in A^{p}$ for all $p<2 / \alpha$.

Lemma 3. For some $p, 1 \leqq p \leqq 2$, suppose that $\sum_{N=1}^{\infty} N^{-p} S_{N}^{(p)}<\infty$ and $N^{1-p} S_{N}^{(p)}=O(1)$. Then $f \in A^{p^{\prime}}, 1 / p+1 / p^{\prime}=1$.

Lemma 1 is proved by an application of Jensen's theorem. Lemmas 2 and 3 follow from corresponding coefficient conditions, after a summation by parts. In particular, Lemma 3 is a consequence of the fact that

AMS (MOS) subject classifications (1970). Primary 30A04; Secondary 30A78.

Key words and phrases. Bergman spaces, $A^{p}$ spaces, $A^{p}$ zero sets, $H^{\nu}$ zero sets. 
$\sum\left|a_{N}\right| N \mid N<\infty$ implies $f \in A^{\infty}$, and that $\sum\left|a_{N} / N\right|^{2} N<\infty$ implies $f \in A^{2}$. One merely applies the Riesz interpolation theorem and summation by parts to obtain the result.

THEOREM 1. Let $0<p<q \leqq \infty$. Then there exists an $A^{p}$ zero set which is not an $A^{q}$ zero set.

SKETCH OF PROOF. Let $f(z)=\prod_{k=0}^{\infty} 1+u z^{b^{k}}$, where $b$ is an integer greater than 2 , and $u$ is a positive constant. Using the notation of the lemmas, one verifies that:

(1) Every partial product for $f(z)$ is a partial sum of its Taylor series.

(2) If $N=\sum_{k=0}^{s-1} b^{k}, S_{N}^{(p)}=\left(1+u^{p}\right)^{s}$.

(3) If $u>1$, if $N=\sum_{k=0}^{s-1} b^{k}$, and if $\left\{z_{i}\right\}$ are the ordered zeros of $f$, then $\prod_{i=1}^{N} 1 /\left|z_{i}\right|=u^{s}$.

From these facts, and from Lemmas 1, 2 and 3, we conclude that:

(4) If $b \leqq 1+u^{2}$, then $f \in A^{p}$ for all $p<2 \log b / \log \left(1+u^{2}\right)$. (Also, in this case, $f \notin A^{2}$.)

(5) If $1+u^{s} \leqq b^{s-1}$ for some $s, 1<s \leqq 2, f \in A^{p}$ for all $p<s^{\prime}$, where $1 / s+1 / s^{\prime}=1$.

(6) If $u>1$, the zero set of $f$ is not the zero set of any function in $A^{q}$ for $q>\log b / \log u$.

An examination of (4), (5) and (6) shows that if $0<p<q \leqq \infty, u$ and $b$ may always be chosen to yield a function $f$ in $A^{p}$ whose zero set is not an $A^{q}$ zero set.

THEOREM 2. For $0<p<\infty$, the union of two $A^{p}$ zero sets is not in general an $A^{p}$ zero set.

To prove Theorem 2, we choose one of the functions $f \in A^{p}$ constructed in Theorem 1, with the parameter $u>1$. We choose a positive integer $N$ and require that each zero of $f$ be repeated $N$ times. For $N$ sufficiently large we obtain a sequence which, by Lemma 1 , cannot be an $A^{p}$ zero set.

We state two corollaries to the above theorems, both of which again contrast sharply with $H^{p}$ theory.

COROLlaRY (TO THEOREM 1). It is not possible to represent an arbitrary $A^{1}$ function as the product of two functions in $A^{2}$, one of them nonvanishing.

Corollary (то TheOREM 2). Consider the operator $M_{z}$ of multiplication by $z$ on $A^{2}$ (a weighted unilateral shift). There exist two nontrivial closed invariant subspaces of $M_{z}$ whose intersection is trivial.

\footnotetext{
Department of Mathematics, University of Michigan, Ann Arbor, Michigan 48104
} 